\title{
Model Reduction of Electric Rotors Subjected to PWM Excitation for Structural Dynamics Design
}

\author{
Margaux TOPENOT ${ }^{1,2}$, Gaël CHEVALLIER ${ }^{1}$, Morvan OUISSE $^{1}$, Damien VAILLANT ${ }^{2}$ \\ ${ }^{1}$ Univ. Bourgogne Franche-Comté, FEMTO-ST Institute, \\ Department of Applied Mechanics \\ 24 Rue de l'Épitaphe, 25000 Besancon, France \\ ${ }^{2}$ Alstom Transport, \\ 7 Avenue du Maréchal de Lattre de Tassigny, 25290 Ornans, France
}

\begin{abstract}
Rotors of asynchronous machines can be subjected to risk of failure due to vibratory fatigue. This is caused by the way electric motors are powered. Pulse Width Modulation (PWM) is the control strategy of the traction chain. This signal is composed by a fundamental and numerous harmonics of voltage and current that induce harmonics on the torque signal resulting in huge torque oscillations. It can lead to repeated torsional resonance when coincidences occur. This can induce severe damages and even lead to rupture if electric excitations are not taken into account at the design stage. In this work, a magnetic finite element model is built by using Fourier decomposition in order to take into account harmonics due to PWM. Pressures exported from this model are used as inputs for mechanical FEM. A mechanical reduced order model is also proposed in order to compute stress in rotating part. This second model allows to reduce time computation and then to consider several operating points to build a complete speed up. A correlation is performed between these two models and rotating tests in order to discuss the relevance of these approaches to design rotor parts.
\end{abstract}

Keywords: Electric motor, vibratory fatigue, Pulse Width Modulation, Structural dynamic design, model reduction.

\section{Introduction and state of the art}

Trains are set into motion through electrical energy. From a theoretical point of view, electric machines can be driven by sine wave signals but practically, due to signal synthesis issues and power limitations, motors are driven using Pulse Width Modulation (PWM). This allows to manage easily the variation of the rotating speed. The PWM is based on a fundamental frequency equal to the sine signal frequency. However, PWM generates small-amplitude oscillations at frequencies corresponding to the harmonics, inducing vibrations and spurious mechanical stress. Figure 1 illustrates the difference between PWM and sine waveform signals. This results in torque oscillations over a wide frequency range and possibly large amplitudes, sometimes leading to the rupture of several rotating parts due to resonance [1].

Wachel plots interference diagram in 1993 in order to check for coincidence of the torsional mode with critical operating speed [2]. Song-Manguelle proves in [3] and Feese in [4] that the variable frequency drive leads to shaft failures due to pulsating torque. Other rotating parts can be subjected to failures like fans [5] [6]. The authors of [7] recommend to pay attention of the drive's voltage spectrum to design mechanical parts. Electromagnetic and mechanical phenomenon should be taken into account, this is why several authors propose to couple the two physics : Hallal and Pellerey propose a weakly coupled methodology in [8] and [9]. As Hallal, Dupont proposes in [10] a methodology to project magnetic load on mechanical mesh. Journeaux exposes in [11] the advantages and the drawbacks of weak and strong coupling. Delforge alerts on the huge computational time to perform such a study in [12]. Van der Giet and al. make the comparison between 2D and 3D coupled electromagnetic and structural dynamic simulation in [13]. From the mechanical point of view, the rotor is a complex assembly. Its modeling is subjected to various assumptions. In particular, the stack lamination 
is relatively hard to model. Millithaler proposes in [14] a methodology to model laminated structures. An identification methodology is proposed by Mogenier in [15] to get material parameters in rotor subjected to bending modes.

The aim of this study is to propose and compare rotors dynamic models able to capture the resonance phenomenon due to PWM excitation. Two models are proposed: the first one consists in weakly coupling magnetic and mechanical finite elements models by using Fourier expansion in order to take into account harmonics due to PWM whereas the second one uses reduced order modelling in order to obtain the structural response faster. The scale of refinement which is necessary to describe the problem with a good accuracy will be discussed after the presentation of the results. Rotating tests have been performed in order to measure the strain in rotating parts for a fixe speed that corresponds to the resonance speed. Figure 2 shows a block diagram that explains how models is compared to reference data.

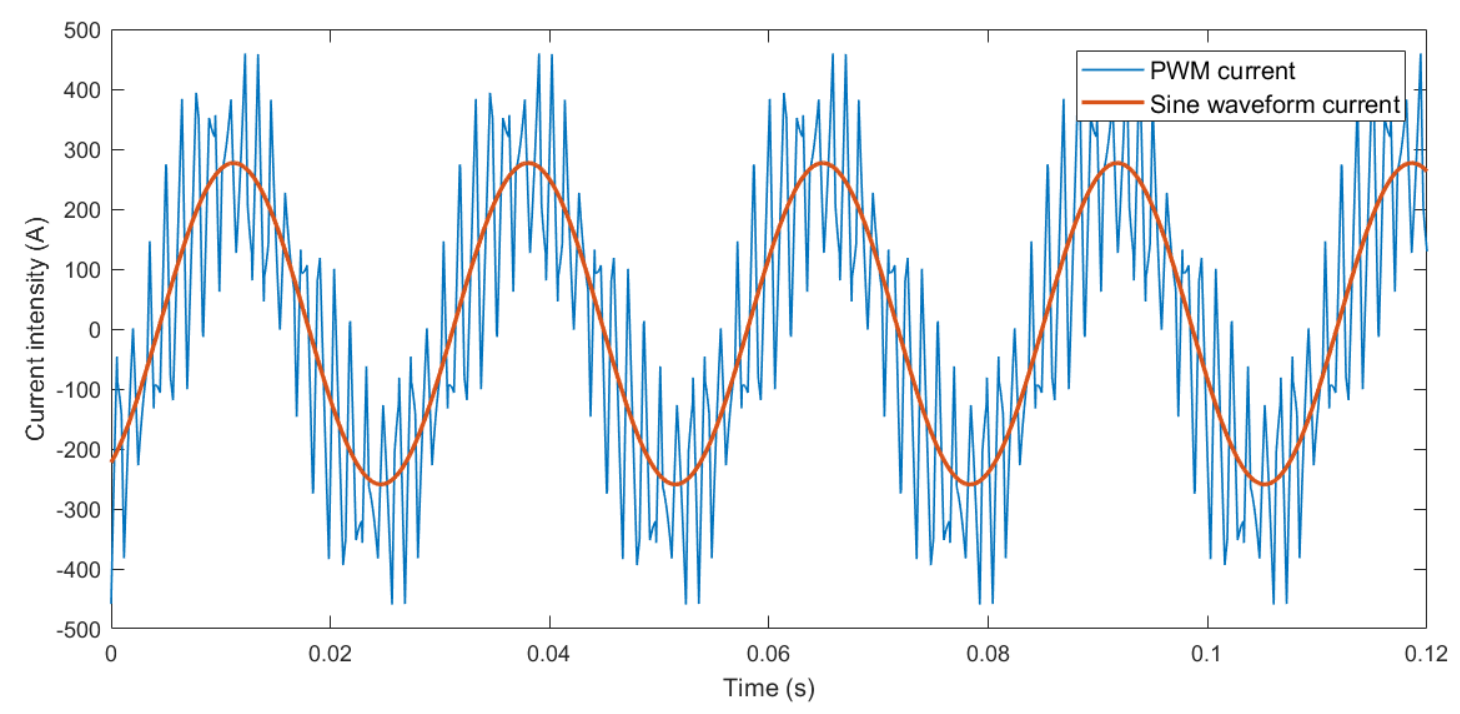

Figure 1: Electric current intensity waveform with PWM or sine

\section{PWM synthesis and stress analysis}

Electro-technical engineers are responsible for the PWM synthesis in the inverter in order to power trains. Several strategies are availables for signal generation [16] according to industrial requirements. This mission requires to wisely choose the way that impulses are created by avoiding losses and increasing performance. It can directly impact the traction chain but also the dynamic behavior of electric rotors. Among all the strategies, asynchronous, synchronous and space vector modulation are the most common. For the two first one, carrier signal is compared to a reference sine waveform signal. This comparison leads to the opening or the closing of switches (IGBT in the inverter). The difference between both lies in the ratio of carrier frequency and the sine frequency. Space vector modulation is a really interesting way to get PWM signals. Indeed, this strategy allows to choose the switching angles (corresponding to switching times). This means that it is possible to change harmonics frequencies in the spectrum of the signal. Due to the performance requirements, it is difficult to completly avoid harmonics but a good knowledge of the studied structure can help to avoid structural dynamic problems.

In this paper, the electromagnetic part of the motor is modeled by an equivalent electric circuit with voltage drive. The voltage source corresponds to the sum of the harmonics of the PWM signals by using Fourier expansion. Authors alert on the limitation of the number of variables that can be set and therefore on the limitation of the number of harmonics taken into account in commercial calculation codes. In general, the carrier frequency is really high compared to frequencies of interest for structural dynamic design. It means that filters can be applied to lower the number of harmonics, but the user must be aware that the reconstructed signal is changed. In addition, if no filter is applied, the maximal frequency is the carrier frequency and then it could lead to very small time steps and then to huge computational time and results files. 
A 3D mechanical finite element model of an electric rotor is considered as reference. The torque provided by the electromagnetic simulation is applied at the rotor center and is also used as load for a reduced order model (see figure 2).

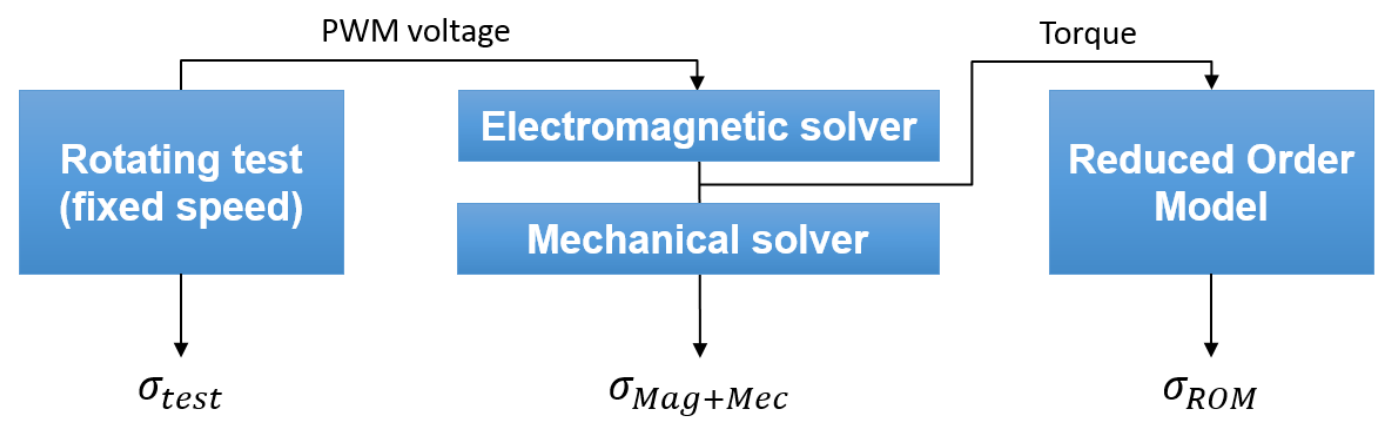

Figure 2: Block diagram illustrating the methodology

An electromechanical lumped model has been published by Bruzzese in 2016 [17]. He managed to define an electrotechnical model by solving equivalent electrical circuit and applying magnetic torque on a simplified mechanical model composed by masses, springs and dampers. Inertia and stiffness matrices were evaluated from a lumped representation. Here, the mechanical reduced order model is built from the modal basis and nodal coordinates of sections coming from the 3D FEM in order to have a more accurate mechanical representation of the real rotor compared to the work proposed in [17]. The key of such a model is the transformation matrix that allows the reduction. Starting from a full 3D model composed of thousands of degrees of freedom, the model is reduced to only nine rotational degrees of freedom. This work being focused on torsion behavior of the electric machine, only rotation degrees of freedom around motor axis are considered. The rotor is decomposed in nine sections as shown in figure 3. The reduction matrix is built by modal projection using the link between displacement and rotational degrees of freedom of rotor sections.

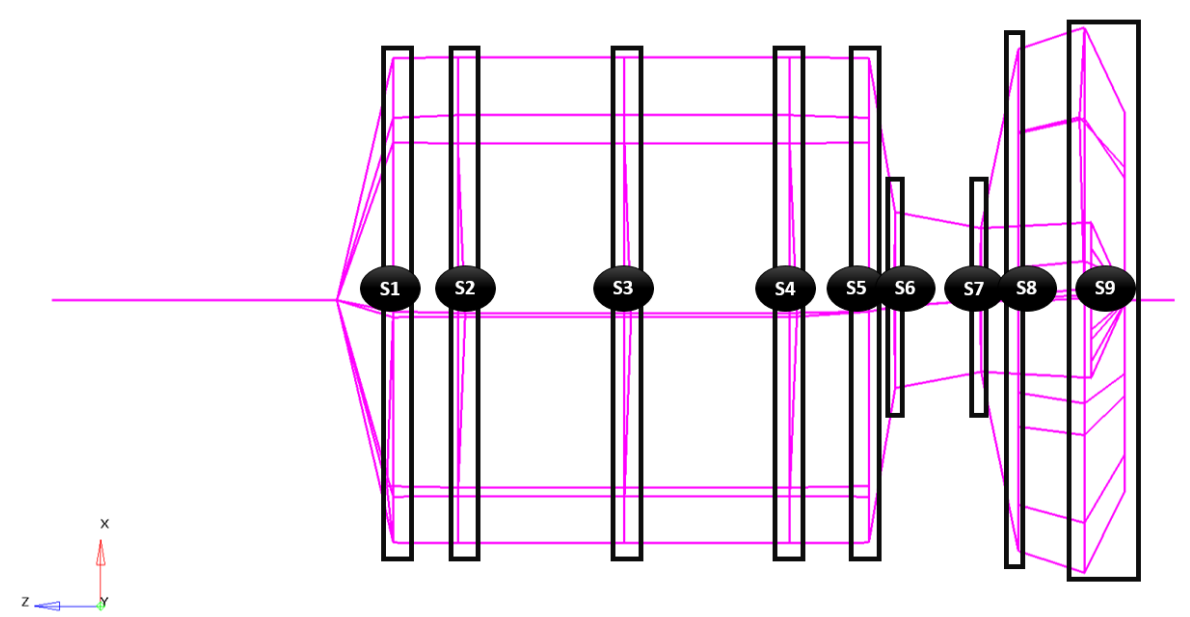

Figure 3: Rotor divided in 9 sections

Writted on the modal basis, the dynamic equation in generalized coordinates $\{q\}$ is

$$
\{\ddot{q}\}+[\Delta]\{\dot{q}\}+[\Lambda]\{q\}=\{f\}
$$

with $[\Delta]=\left[\begin{array}{lll}\ddots & & \\ & 2 \xi_{i} \omega_{i} & \\ & & \ddots\end{array}\right], \xi_{i}$ being the modal damping ratio, $[\Lambda]=[\Phi]^{T}[K][\Phi]=\left[\begin{array}{lll}\ddots & & \\ & \omega_{i}^{2} & \\ & & \ddots\end{array}\right],[\Phi]$ being the modal basis and $[K]$ the stiffness matrix, $\{f\}=[\Phi]^{T}\{F\},\{F\}$ being the load vector. The mechanical 
stresses are obtained by using the modal stresses :

$$
\{\sigma\}=\left[\Phi_{\sigma}\right]\{q\}
$$

\section{Results}

Load excitations and modal behavior should be mastered to predict the dynamic response of the rotor. Figure 2 shows what quantities should be compared. The torque signal and the spectrum exported from electromagnetic simulation is illustrated in figure 4 . This is the input load for mechanical computation.
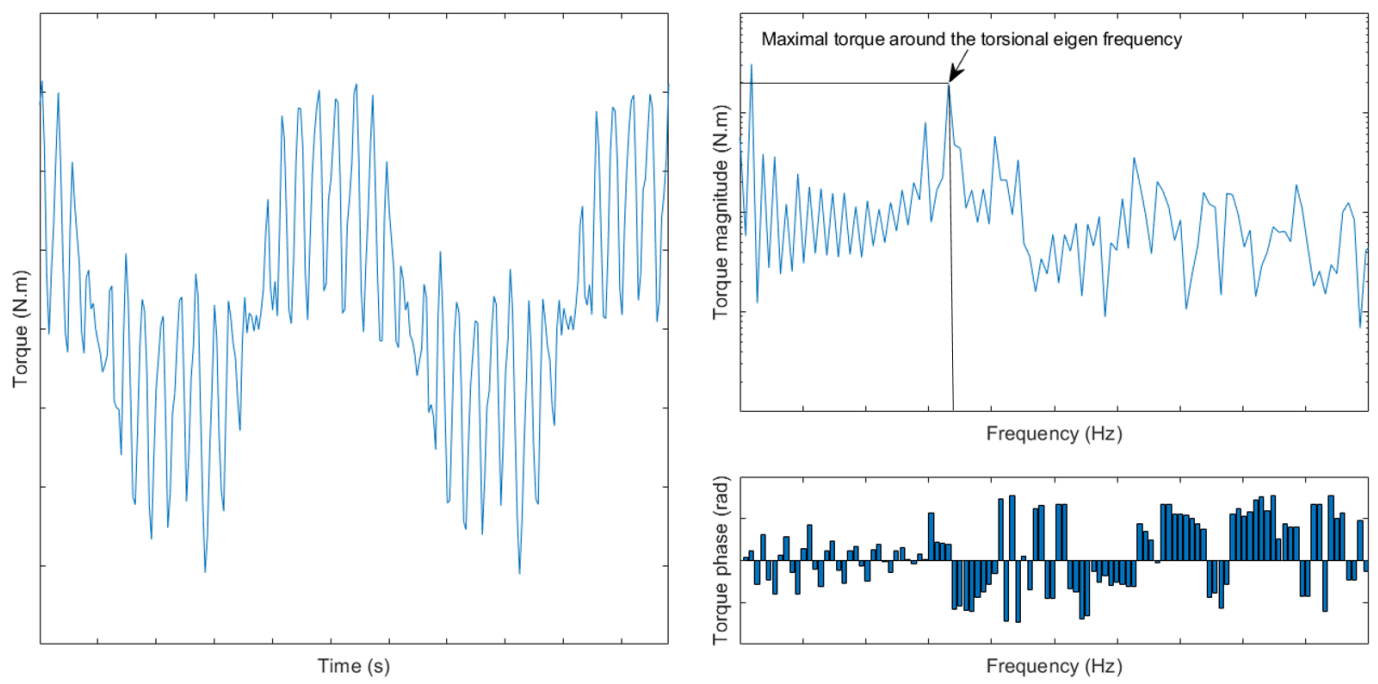

Figure 4: Torque oscillations due to PWM (left side) and FFT of this signal (right side)

Numerically, high torque harmonics are close to the natural frequency of the rotor. But experimentaly, the torque has been measured with a device that allows only to measure a signal until $250 \mathrm{~Hz}$. This means that torque spectrums can not be compared. However, mean values of experimental and numerical torque can be compared in order to validate the simulation methodology. The 5\% gap between the two indicates that the model is representative of the test conditions.

A coincidence between the load harmonics and the natural frequency of the torsion mode is observed in the test data. In order to reproduce this effect with models, the maximal torque value in the spectrum has been spread around the torsional eigen frequency in numerical models. This spreading is necessary because a small error in models can cause the absence of the coincidence. It results in $4 \%$ of relative error on the maximal stress at the torsional resonance between the full model and the reduced order model. This indicates that reduced order model is efficient to describe the behavior of the structure and allows to consider a full speed up.

The computation of the modal basis takes 8 minutes on a calculator with $16 \mathrm{CPU}, 3.2 \mathrm{GHz}$ and $225692 \mathrm{MB}$ RAM and then the calculation of rotation and stresses thanks to reduced model takes 2 minutes. In comparison, the magnetic computation with magnetic solver takes 5 hours on a laptop with $8 \mathrm{CPU}, 2.7 \mathrm{GHz}$ and $16 \mathrm{Go}$ RAM and then the mechanical computation with mechanical FE solver takes 2 hours on the calculator.

The figure 5 illustrates qualitatively the comparison of the measured waterfall of the stress (left side) and the computed one (right side). Resonance clearly appears when load harmonics cross the eigen frequency.

It remains to calibrate the model to fit the rotating test data. Several aspects should be taken into account :

- the damping ratio of the rotor has been measured in free-free condition and may be very different in the full motor in rotating condition ;

- even if the constant value of the computed torque fits well the average value of the experimental mechanical torque, the computed torque is the electromagnetic torque and due to losses, it might not be identical to the mechanical torque. The inertia of the rotor might filter the torque ; 

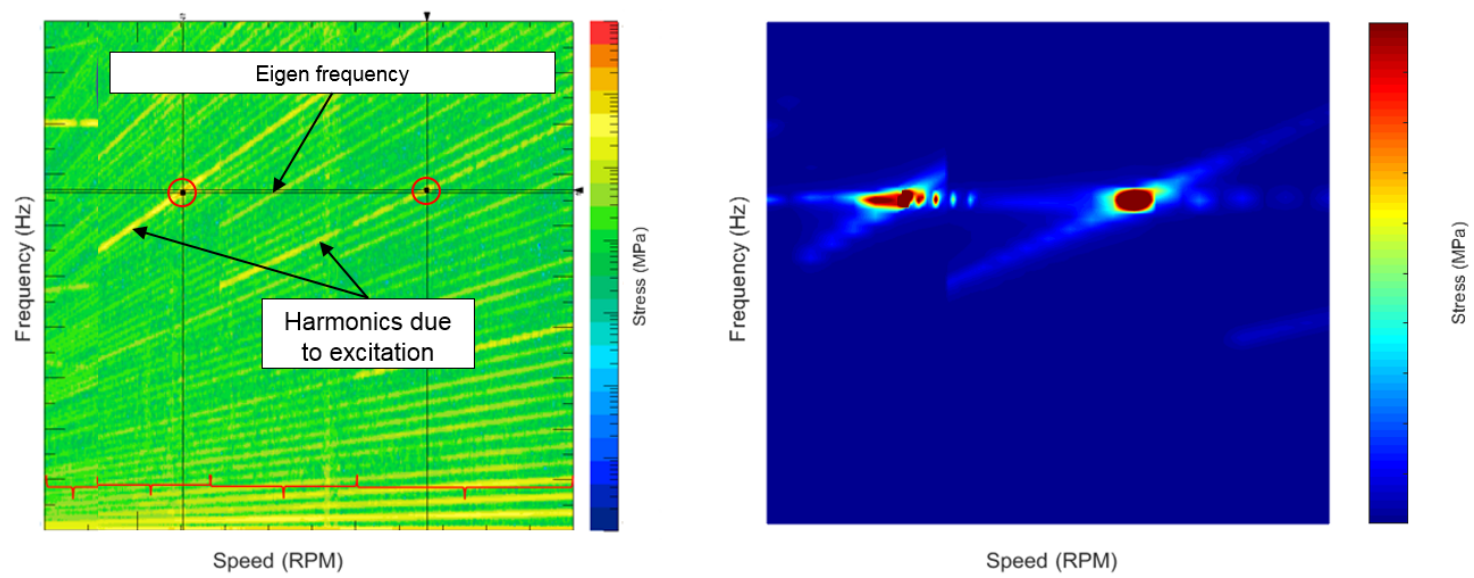

Figure 5: Comparison of stress waterfall obtained by measurement (left side) and by computation with the reduced order model (right side)

- uncertainties about the location and the orientation of the gauges can lead to a bad correlation between measured and computed strain ;

- during tests, the speed is oscillating and it can lead to a rotor that is not excited exactly at the resonance, or the slip changes during tests due to temperature that not allows to stay at the resonance for a long time.

All these aspects can explain differences between tests and models. Modelling assumptions must be carefully chosen.

\section{Conclusion}

This paper compares two computational methodologies to investigate the vibratory response of electric rotors subjected to PWM excitation. The first one combines two Finite Elements Models in order to solve the multiphysic problem. The torque computed from the magnetic solver with test PWM signal is the input of the computation of the structural response to determine the mechanical stresses. Secondly, a model reduction is proposed to fasten the computation time during design phase. The $4 \%$ difference on maximal stress at the torsional resonance between the full model and the reduced order model allows to conclude that the reduction is accurate enough and efficient to compute the stress along a speed up. However, it remains to calibrate models to tests data. Future work will focus on the understanding of phenomenon and assumptions to be choose to fit tests data. Model updating will allow a better prediction of rotor vibratory response.

\section{References}

[1] C. Bruzzese, A. Tessarolo and E. Santini, (2016, September). "Failure root-cause analysis of end-ring torsional resonances and bar breakages in fabricated-cage induction motors". In 2016 XXII International Conference on Electrical Machines (ICEM) (pp. 2251-2258). IEEE.

[2] J. C. Wachel and F. R. Szenasi, "Analysis of Torsional Vibrations in Rotating Machinery", 1993. Proceedings of the 22th Turbomachinery symposium. Texas A \& M University. Turbomachinery Laboratories.

[3] J. Song-Manguelle, S. Schroder, T. Geyer, G. Ekemb, and J.-M. Nyobe-Yome, "Prediction of Mechanical Shaft Failures Due to Pulsating Torques of Variable-Frequency Drives," IEEE Transactions on Industry Applications, vol. 46, pp. 1979-1988, Sept. 2010.

[4] T. Feese and R. Maxfield, "Torsional vibration problem with motor/ID fan system due to PWM variable frequency drive", 2008. Proceedings of the 37th Turbomachinery Symposium. 
[5] S. Kreitzer, J. Obermeyer, and R. Mistry, "The Effects of Structural and Localized Resonances on Induction Motor Performance," IEEE Transactions on Industry Applications, vol. 44, pp. 1367-1375, Sept. 2008.

[6] J. H. Holdrege, W. Subler, and W. E. Frasier, "AC Induction Motor Torsional Vibration Consideration - A Case Study,” IEEE Transactions on Industry Applications, vol. IA-19, pp. 68-73, Jan. 1983.

[7] R. J. Kerkman, J. Theisen, and K. Shah, "PWM inverters producing torsional components in AC motors," in 2008 55th IEEE Petroleum and Chemical Industry Technical Conference, (Cincinnati, OH), pp. 1-9, IEEE, Sept. 2008.

[8] J. Hallal, Études des vibrations d'origine électromagnétique d'une machine électrique: conception optimisée et variabilité du comportement vibratoire. 2014. $\mathrm{PhD}$ thesis. Compiègne.

[9] P. Pellerey, V. Lanfranchi, and G. Friedrich, "Coupled numerical simulation between electromagnetic and structural models. Influence of the supply harmonics for synchronous machine vibrations", IEEE Transactions on Magnetics 48 (2012) 983-986.

[10] J.-B. Dupont, P. Bouvet, and L. Humbert, "Vibroacoustic simulation of an electric motor methodology and focus on the structural FEM representativity", International Conference on Electrical Machines, Marseille, 2012, pp. 1-7.

[11] A. Journeaux, Modélisation multi-physique en génie électrique. Application au couplage magnétothermo-mécanique. PhD thesis, 2013. Université Paris-Sud.

[12] C. Delforge and B. Lemaire-Semail, "Induction machine modeling using finite element and permeance network methods," IEEE Transactions on Magnetics, vol. 31, pp. 2092-2095, May 1995.

[13] M. Van der Giet, C. Schlensok, B. Schmulling, and K. Hameyer, "Comparison of 2-D and 3-D Coupled Electromagnetic and Structure-Dynamic Simulation of Electrical Machines," IEEE Transactions on Magnetics, vol. 44, pp. 1594-1597, June 2008.

[14] P. Millithaler, E. Sadoulet-Reboul, M. Ouisse, J.B. Dupont, N. Bouhaddi, "Structural dynamics of electric machine stators: Modelling guidelines and identification of three-dimensional equivalent material properties for multi-layered orthotropic laminates", J. Sound Vib. 348 (2015) 185-205.

[15] G. Mogenier, Identification et prévision du comportement dynamique des rotors feuilletés en flexion. $\mathrm{PhD}$ thesis, 2011. Institut National des Sciences Appliquées de Lyon.

[16] S. Bowes and R. Clements, "Computer-aided design of PWM inverter systems," IEE Proceedings B Electric Power Applications, vol. 129, no. 1, p. 1, 1982.

[17] C. Bruzzese, \& E. Santini, "Electromechanical modeling of a railway induction drive prone to cage vibration failures." IECON 2016-42nd Annual Conference of the IEEE Industrial Electronics Society. IEEE, 2016 